ciple of surgery must be patent to all. An abscess should not only be opened, but should be kept open until the pyogenic process ceases. It is a fallacious doctrine that air can or should be excluded from the middle ear in this manner. Air will gain access to this cavity through the Eustachian tube and furnish pathogenic germs. Free drainage should be maintained through the canal built by Nature, in order to avoid a complication of the existing trouble with mastoid disease. Moreover, it is problematical whether one ever thoroughly cleanses the middle ear of pus by this dry method, even if the perforation be an unusually large one. The cotton is not likely to enter the tympanic cavity and it is not desirable to have it for there would be danger of entangling the ossicles in its meshes and dislocating or removing them.

The most rational and successful treatment $I$ have tried is the following: Cleansing the external meatus and middle ear thoroughly with injections of a r-10,000 solution of mercuric bichloride; inflation by the Politzer method, or catheterization; iodine vapor if stimulation is required; drying the part with absorbent cotton and dusting them with finely pulverized boracic àcid containing $1 / 2$ of $I$ per cent. of mercuric bichloride, or if this should cause any unpleasant sensation, iodoform or boracic acid may be substituted.

Hydrogen peroxide is valuable for cleansing the ear when there is a large amount of debris present in the form of pus mixed with epithelial scales, or cheesy concretions. In addition to its excellent mechanical effect due to effervescence, the oxygen liberated destroys bacteria. The latter result is also effected by the sublimate solution which in one-half the strength mentioned will destroy bacteria in ten minutes. The inflation ought to expel the fluid contents of the middle ear. In cases of brief duration iodine is not required, but in very old cases, when the vital forces seem to have lost their powers of recuperation and resistance to pathogenic germs, tissue changes - the process of absorption and nutrition-may be favorably influenced by the judicious use of iodine vapor. Drying the parts before dusting them with the powder leaves the patient more comfortable than the chilling effect of evaporation does. The powder when slightly wet becomes hard and produces a feeling of stiffness, and sometimes of soreness. Then if the powder be left dry we are enabled to determine at once when the discharge ceases.

It is not necessary to fill the ear with powder. If enough be insufflated to barely cover the suppurating membrane, all is accomplished that can be expected from the remedy, and the functions of the ear are not materially interfered with-an important consid. eration with many patients. This treatment leaves no obstruction to free drainage, and in no manner invites mastoid trouble. When the disease has just passed from the acute to the chronic stage the boracic acid powder had better be used without the bichloride, for the latter may cause some disagreeable crackling sensations and even pain. But in the strength mentioned it is not likely to do so unless there is considerable inflammation.
At the meeting of the Illinois State Medical Society, held in this city three weeks ago, the efficacy of iodoform as an antiseptic was called in question. While the experiments of Heyn and Rovsing, of Copenhagen, show that iodoform is inert in the presence of bacteria while the remedy remains in a dry state, de Ruyter has proved that iodoform in the presence of the fluids of suppurating surfaces undergoes chemical decomposition during which new iodine conpounds are formed. The splitting up of the iodoform, its partial solution and absorption, resulted in the destruction of the ptomaines, the product of pathogenic micro-organisms, and hence the arrest of pathological metamorphosis. The laboratory thus confirms the practical conclusions which years of experience have forced upon the profession. In conclusion, let me add in support of the method here outlined, that no routine treatment has ever yielded the uniformly satisfactory results in my hands that this has. Were there time to enter into more minute details it would be interesting to consider the variations of treatment required by necrosis, etc., but that would extend beyond the scope of this paper. Numerous illustrative cases might be adduced from my records, but I will mention but one that I have now under observation, in which the hearing was nil from chronic non-suppurative inflammation of the left middle ear, and the voice could be heard only by shouting in the right ear, in which chronic suppurative inflammation had existed for over forty-nine years. I removed from both nostrils large polypi, which had prevented nasal respiration for twelve years. The treatment I have described stopped the discharge in four days, and subsequent catheterization, etc., restored some hearing in the left ear, and so improved the right as to render conversation audible at a distance of fifteen inches.

719 West Adams Street.

\section{RESECTION OF THE INTESTINE FOR FECAL FISTULA.}

$$
\begin{aligned}
& \text { Read before the Chicago Medical Society, October 3, } 8887 . \\
& \text { BY F. HENROTIN, M.D., } \\
& \text { of CHICAGo, ILL. }
\end{aligned}
$$

About five weeks ago I was called to Pullman, in this State, to see a patient. On my arrival I found the patient affected with frecal fistula. The history of the case was this: The woman had been affected with an obstruction of the bowels about fifteen days before I saw her. The obstruction was evidently in the left inguinal region, as a mass was found there, which the physician in cliarge tried to reduce. Fail ing in reducing it, she called in consultation a physician from Chicago, who went out there, and by dint of manipulation they succeeded in reducing this hernia. Immediately succeeding the reduction of the mass in the groin, the patient complained of a great deal of pain, and a truss was fitted over the place where the hernia had existed. This truss being somewhat tight gave rise to a great deal of pain, and after two or three attempts at periodical times to wear the truss it was laid aside. The parts 
by this time were considerably swollen and required attention. In four or five days an abscess over the part had formed. The pus was evacuated, and within forty-eight hours there was seen a liquid substance oozing from the wound, and this developed into a well marked fæcal fistula. (I should say that this was a femoral hernia on the left side.) The fistulous opening was, at the time I saw the patient, very large and at the outer part would easily admit the thumb. On examination I found the woman very much emaciated, thin and extremely weak. She had then had this fistulous opening running about eight days; the parts around the opening were excoriated and the edges of the wound were in a gangrenous condition. The discharge from it was excessive, and consisted of a greenish fluid exuding in large quantities, and mixed with this was a yellowish emulsive substance, having a slight fæcal odor.

The weakness of the patient and her general condition, as well as the appearance of the wound, made me decide upon an operation. Having given the matter careful consideration I operated the second day following. There was no opportunity to do the operation under antiseptic precautions. The surroundings were not the best for such an operation, but as far as it lay in my power antiseptic precautions were thoroughly carried out. The instruments were all very carefully gone over beforehand, ard the sponges and all material used was thoroughly and carefully prepared. I decided upon simply making an incision in the linea alba of the lower abdomen, and taking out and detaching the bowel.

I made an incision about three and one-half inches in length so as to get sufficient room, and entering the abdomen I slipped a large sponge into the opening, so that the end was immediately beneath the attachment of the bowel at the opening; I also packed in two or three little sponges at the side of this long sponge, in order to protect the parts as much as possible. The large sponge reached from a little below the fistulous opening to the opening in the abdomen, so as to cover the parts thoroughly. I then detached the ring of the intestine by slipping a finger around it and gradually working it apart, and so was able to grasp the intestine and bring it to the surface. To make sure that the intestine did not slip, I had introduced a female catheter through the external opening four or five inches into the bowel, so as to be perfectly certain of bringing the parts to the opening without delay. Everything worked very satisfactorily, and the bowel was brought to the surface, and outside upon the abdomen in a very few moments. The only part in which there was any difficulty was in detaching the bowel from the opening. Never having tried the procedure before, I was surprised at the amount of force necessary to detach it. When I brought the bowel to the surface I packed sponges around it, and held the edges of the wound together in such a way that I knew that there was no possibility of further infection during the fixing up of the bowel. Much to my chagrin, when I had brought the bowel to the surface, I found there was a very large and jagged opening in the bowel, and that the efforts at detachment had served it almost down to the mesentery.

There apparently remained nothing to do but to take away the part, resect the intestine entirely, which I immediately did. Taking the bowel up carefully (with these prepuce forceps) on each side, about three-fourths of an inch beyond the furthest point of the tear, I cut the bowel off square on the side of the tear, leaving one-fourth of an inch margin. These forceps answered the purpose admirably, each blade being covered with a soft rubber so as to leave no impression on the bowel. I took them off once or twice during the process of stitching, and found there was not too much pressure being used, not enough to wound or injure the bowel. Having cut off the bowel down to the mesentery I thought it best not to injure the mesentery, which proved, I think, a great element in the success of the case, and which has been further proved to my own satisfaction to be the proper way of procedure. When I reached the mesentery I simply cut right straight across along the edge of the mesentery, and left it as nearly whole as I could, cutting directly in a line between the bowel and mesentery. I then stitched up the intestine. After stitching up the bowel and fixing up the mesentery to my satisfaction, I returned the bowel to the abdominal cavity, and looked for any debris or blood, but not finding any stitched up the abdominal wall tightly. I had the long narrow sponge left from one opening to the other, and apparently not a drop of oozing had taken place in the abdomen. I replaced this long sponge with a flatter one before stitching the abdomen, so as to protect it from any bleeding stitch holes, and in a short time had the wound closed, and had the satisfaction of knowing that there was no blood in the abdominal cavity.

As regards the original opening, I simply scraped it off under Poupart's ligament, refreshed it, and introduced through that opening my drainage tube, closing the central wound entirely. I also introduced a second tube into a sinus that went up along Poupart's ligament, along the inguinal canal.

The case did remarkably well. At no time was there any temperature beyond a few fractions of a degree above Ioo. She never even vomited. There was no tympanitis, no bad symptom whatever. The patient is now, after several weeks, doing quite well. She was 59 years old and extremely delicate, but she is apparently very nearly in her normal health except that she has some colicky pains and occasionally a loose stool; whether this is due to a stricture and may prove permanent in character, I cannot say.

The after treatment needs no consideration. The dressing put on was merely the ordinary dressing of laparotomy after àn ovariotomy-dry iodoform, an antiseptic cotton pad and antiseptic gauze outside. The dressings were only disturbed once every four or five days. There was a little abscess in the abdominal wall, but it gave no trouble and healed in a few days.

The particular point I wish to bring to your attention is the propriety of operation. The first item 
of importance, which was very evident, was the extreme debility of the patient. She was naturally a very weak woman, was 59 years of age; and whatever was to be done had evidently to be done soon. Physicians who had seen the case and who assisted me at the operation, were also of that opinion, and they were possibly better able to judge than I was as they had watched the case from the first. The patient was evidently failing, the pulse and general appearance indicated a great deal of weak. ness, and in addition was this constant drain. cannot tell the amount because I did not see the patient long enough, but it was considerable; the cloths and cotton padding, and dressings that were put on had to be changed several times a day, and when the abdomen was pressed above the wound this greenish fluid bubbled up in large quantities, mixed with a yellowish substance which I considered to be chyme, or rather chyle. What else was to be done? Of course we know that in a fistulous opening of that kind it is an ordinary thing to make a plastic operation of some kind, but in this woman such an operation would have failed on account of the amount of fluid and the character of the opening, its edges being gangrenous, a pulpy greenishyellow. The only way in which that patient could have been kept alive would have been to have given her food by the mouth, and tried to find the lower opening, injecting food in that portion of the bowel, and it would have been very hard to tell which was the lower opening. The anatomy of the abdominal organs is such that we cannot always tell whether we are going up or down under such circumstances. But this case had to be taken care of, and something done at once. The character of the fluid denoted that the opening was extremely high up; there was no dark substance, nothing that would represent fæcal matter, no appearance to indicate that it was low down, and the constant drain and the amount of fluid indicated that it was high up, because we know the villi of the intestines and lacteals take up the fluid as it goes along, and the lower down we get in to the bowel the more the fluid disappears. For these various reasons it seemed to me that the one thing to do was an immediate operation; that in view of the weakness of the patient, the character of the fluid and the evident high situation of the fistula, any other procedure would be followed by failure.

The reason of the resection of the bowel is evident to you all, on viewing the specimen. It certainly would not be very good policy to try and patch up a bowel torn up in that way. As regards the way in which the operation and the stitching of the bowel was done and the way in which the mesentery was treated: In taking the bowel out I found that the serous coat was extremely soft and pulpy, and I soon found, on trying to put a stitch through, that the least little pull would tear through the serous coat, and I really believe that a large portion of cases of resection of the bowel, where the stitches are taken as much as possible in the serous membrane, are very liable to fail on that account. The way I stitched up the bowel was this: I put one row of stitches made of silk in the whole thickness of the bowel, the three coats. I started in on the edge on one side and then came out so as to bring my free ends on the inside of the bowel. I then tied. This was a very easy matter when the two portions of the bowel were held up by the forceps, and after thorough cleansing the two parallel edges were sewed together, each stitch being put so that it .would fall inside when the bowel was brought around. The stitches were placed a little over a sixth of an inch apart, and I had no difficulty in going around the bowel in that way, always going through each of the three coats. When I got down to the last two stitches I began on the outside and brought the needle through what was left of the ununited inside of the bowel, then started another needle through the same way and brought them both out and tied. The last stitch I put in the same way, bringing out the needles from each side through a little crevice and tied as close as I could, and then it was easy to take the knot and insinuate it between the edges of the last two stitches, so that it was tied on the inside. I then put in a suture of continuous catgut stitched on the outside serous coat only. After each stitch I had my assistant draw the catgut snug to hold the ground I had gone over, and so managed to surround the whole bowel without difficulty.

As regards the mesentery, I did not interfere with it, but treated it in this way. Here we have a section, this being the bowel above and this the mesentery below. When I got down to this I stitched my mesentery in this way, not going through to this side so as to interfere with the circulation in this direction, and to make a $\mathrm{V}$ and hold it together in this way. You can see the absolute security which it gives. All authorities agree that interference with the mesentery is what produces the liability to sloughs, and they are extremely careful to tell us to interfere with the mesentery the least we can. Another good reason for this treatment is that when the parts are brought together and the mesentery is not held its movements produce a strain on these two or three lower stitches and cause them to cut through, and at the same time allow fluids to pass out and produce fatal results. So I stitched the mesentery in order not to destroy any of it, and at the same time hold it together so as to make the parts perfectly firm as a support to the bowel.

Another item that may or may not prove of importance was this: In cutting the bowel down on each side, having my clamp on, just before reaching the mesentery I curved my section slightly toward the side of the wound on both sides. It seems to me this is of value, as this is apt to be a weak spot. There being an accumulated amount of tissue here you can with this thickness of tissue take in a stitch on either side and hold them firmly so as to prevent as far as possible any strain at that place. The larger the amount of tissue the firmer the union will be.

One great factor toward the favorable issue of the case was that the woman hardly stirred, and did not vomit throughout the whole operation, and that the bowels at the time were almost absolutely flat. That has a great deal to do with the success of such cases. It is a question whether in a certain proportion of 
cases of hernia of a certain variety it would not be better to operate in this way. About a week ago I was called to see a patient in Lake View who was vomiting and had evident obstruction of the bowels. On examining the patient I found a bunch of something peculiar in the groin in the inguinal canal on the right side. It was evidently something coming from the inside of the abdomen, but I could not make out what it was. It did not have the appearance of bowel and I was loth to manipulate it very much. Yet the anatomical relations were such that it evidently came from there. I tried in every way to reduce it, but without success. I was so certain that it was not the bowel but was omentum that I allowed 48 hours to go by in order to watch the symptoms. At the end of 48 hours, and after various attempts at reduction, on Thursday I cut down on it and found, coming out of a very small opening, a piece of strangulated omentum. The condition of the patient was such that it was with great difficulty that we could stay in the room and operate on account of the stench from his vomiting. He lived a great distance from my home, and possibly I did not operate early enough. During the night the patient had a free stool, the first he had had for a week, the stercoraceous vomiting stopped, and for 48 hours he did well. The patient is alive yet and has a fair show, but the vomiting has recommenced and he does not look very well.

Now, how much of the omentum is injured? I cut off the omentum as close as $I$ could; pulled it out as far as I could before it was cut and tied it at the internal ring, and after tying it carefully with catgut I cut it off and put back the stump. How much of that omentum is gangrenous behind that ligation? Of course the condition of the patient may be due to something else. I introduced the finger right into the abdominal cavity, and there is no greater danger in making the incision in the middle line and stretching the opening from the inside carefully with the finger and pulling out the omentum to see what you are doing. Our President told me that he once tied and cut off an omentum in an umbilical hernia. The patient died and the post-mortem examination showed this omentum stretched and twisted around and strangulating the intestines, and of course the patient was not relieved by the operation. The two cases taken together suggest the propriety in some cases of hernia that have stood for a long time, that were originally acute cases and probably omental, but where, from the length of time the hernia had existed, there was reason to suppose that there was considerable injury to the bowel or omentum, as to whether it would not be the best thing in these cases to open the abdomen carefully in the middle line, because you can reach down in the groin and, after stretching the internal and if necessary the external ring, bring out the strangulated parts and see plainly what is necessary to do.

On examination of the specimen before you one thing is plainly discernible: I was not mistaken as to the locality of the injury. There are no certain ways of always knowing the exact portion of the bowel affected. The lower part of the diodenum does not differ particularly from the upper part of the jejunum, but we know that the higher up we are the greater is the amount of folds of rugæ so-called valvular conniventes that are around the bowel. When I operated on this bowel it was nothing but a mass of corrugated folds on its mucous aspect, and though this specimen has been more or less changed in appearance by being immersed in diluted alcohol, you can see all along on the inner side the rugæ that are still present, showing about the only way that I know of to distinguish the upper part of the small intestine. Of course the anatomical relations of the bowel differ in different persons. However, from its appearance and location, I suspect that this specimen is a portion of the jejunum.

\section{ARTIFICIAL RIPENING OF CATARACTS.}

Read before the Chicago Society of Ophthalmology and Otology. BY BOERNE BETTMAN, M.D.,

of CHICAGo.

This is a subject which has not received the recog. nition from fellow-colleagues that its importance deserves. Only a few articles and comments, scattered here and there, can be found relating to the relief of persons having non-mature cataracts. The third number, Vol. xl, of Knapp's Archives, contains a communication from the able pen of Prof. Forster, of Breslau, entitled "On the Maturity of Cataract." Förster calls attention to the annoyances patients are subjected to who are afflicted with cataracts of slow development. In many persons the process of degeneration extends over a period of months and years, leaving them in a helpless condition, and in many instances obliging them to forego the enjoyments of life and resign life-supporting and profitable positions.

So long as the cararact is not mature, an operation is contraindicated. So long as the cortical substance is still firmly adherent to the capsule, extraction of the lens cannot be performed with perfect safety to the patient, for, as Förster correctly states, the remaining layers of cortical substance will imbibe aqueous humor, swell, and give rise to iritis and other dangerous complications. In order to produce more rapid degeneration of the lens substance, he proposes trituration of the cortex. The procedure consists of gently rubbing the cornea with the blunt end of an instrument, usually a strabismus hook or iridectomy forceps. The pressure exerted upon the cornea is transmitted to the lens, loosening and splitting the still adherent and semi-opaque cortical lens fibres. These, in the course of days and weeks, become perfectly opaque. The object of the operation has been accomplished; the cataract is mature. I was very much impressed by Prof. Förster's article, and resolved to try his method whenever occasion permitted. I have used the expedient quite often, but have modified it to suit my individual tastes. Instead of rubbing the cornea, I bring the pressure to bear directly upon the lens, by pressing against the capsule with a spatula, usually employed 